\title{
Evaluation of Factors Associated with Tongue Coating Status in Elderly with Care Needs
}

\author{
Koichiro Ogami, Takayuki Ueda, Masahiro Ryu, Sayaka Tajima and \\ Kaoru Sakurai
}

Department of Removable Prosthodontics and Gerodontology, Tokyo Dental College, 2-9-18 Kanda-Misakicho, Chiyoda-ku, Tokyo 101-0061, Japan

Received 5 October, 2017/Accepted for publication 4 December, 2017

\begin{abstract}
The tongue surface in older people requiring nursing care is often coated with a layer believed to comprise, keratinized products of degeneration, food residue, bacteria, and leukocytes attached to the tongue papillae. Which factors affect the status of this tongue coating remain to be elucidated, however. The purpose of this study was to evaluate factors related to tongue coating status in older people requiring nursing care. The study participants comprised 41 individuals admitted to special nursing homes for the elderly. After undergoing tooth brushing and tongue cleaning by the facility staff, the participants were required to refrain from oral cleaning until evaluation of tongue coating status, which was performed just after lunch. The moisture level of the tongue surface was measured and total number of oral microbes determined. Food type, care level, and sex were determined by a questionnaire given by the attending nurses. Correlations between the tongue coating status and various factors were analyzed using Spearman's rank correlation coefficient. The participants were divided into 2 groups according to the median value of the tongue coating status. Factors related to tongue coating were analyzed using binomial logistic regression analysis $(\alpha=0.05)$. Tongue coating status showed a significant correlation with the total number of oral microbes on the tongue surface $\left(r_{s}=0.449, p=0.003\right)$, type of food $\left(r_{s}=0.388, p=0.012\right)$, and care level $\left(r_{s}=-0.308\right.$, $p=0.050)$. Logistic regression analysis revealed that the food type of the side dish significantly affected tongue coating status. These results showed a correlation between tongue coating status and type of food of the side dish in older people requiring nursing care.
\end{abstract}

Key words: Tongue coating — Food style — Oral hygiene — Nursing care — Aged

\section{Introduction}

Pneumonia is a major cause of death in older people, and aspiration pneumonia, in particular, occupies a high percentage of lethal pneumonia. Aspiration pneumonia is caused by silent aspiration of saliva containing resident microorganisms of the oral cavity and pharynx. In older people, in particular, the risk of aspiration pneumonia is believed 
to increase as a result of decreased secretion of saliva and inadequate tooth brushing due to a decline motor function in the fingers. Moreover, the state of oral hygiene is likely to deteriorate in older people with a low score for activities of daily living. Plaque contains a high percentage of the bacteria responsible for pneumonia, with a buildup of this reservoir increasing the risk of aspiration pneumonia. Periodontal pathogens, a cause of aspiration pneumonia, were detected frequently on the tongue surface under edentulous conditions $^{16}$. Moreover, the tongue surface is often coated with tongue coating in older people requiring nursing care.

This coating resembles dental plaque, and is believed to comprise keratinized products of degeneration, food residue, bacteria, and leukocytes attached to the tongue papillae. It is significant, because it builds up on the mucosa over a wide area of the oral cavity, including the dorsum of the tongue, from its center to the base, and is observed regardless of age or state of dental caries or periodontal disease $^{2,5,15)}$. Serving as a reservoir for microorganisms, it harbors more than 700 species, and its status has been demonstrated to show a correlation with the total number of anaerobic bacteria on the tongue surface and in the saliva of edentulous people conditions ${ }^{16)}$.

In older people, the most common type of pneumonia is aspiration pneumonia, which is induced by a decline in swallowing function and the cough and swallowing reflexes. Thus, aspiration pneumonia can be caused by silent aspiration of saliva containing microorganisms present in the oral cavity and pharynx. In a 2-year follow-up study of 400 nursing home residents, the incidence of pneumonia was $11 \%$ in those receiving oral hygiene intervention by dentists and dental hygienists and $19 \%$ in those who were not, suggesting that oral hygiene care contributes to prevention of pneumonia ${ }^{17,18)}$. Factors affecting tongue coating status remain to be elucidated, however. The purpose of the present study was to evaluate factors related to tongue coating status in older people with care needs.

\section{Materials and Methods}

The study participants comprised nursing home residents aged 65 years or above. All were receiving professional oral cleaning. Most had removable dentures, but some of them did not use them. Acute oral problems required exclusion from the study. The participants were instructed to refrain from cleaning of the oral cavity following an initial round of tooth brushing and tongue cleaning provided by care givers after breakfast. Tongue coating status was then evaluated after lunch by the method of Shimizu et al (Fig. 1 $)^{12}$. Moisture level on the tongue surface, the total number of oral microbes on the tongue surface, type of food, care level, and sex were evaluated as items related to tongue coating status (Table 1). The moisture level on the tongue surface was measured using an oral moisture meter (Mucus ${ }^{\circledast}$; Life, Saitama, Japan). To perform these measurements, the sensor of this device was brought into vertical contact with the surface of the tongue at $10 \mathrm{~mm}$ from its tip. Each measurement was performed 3 times and the median value used for the analysis. It is difficult to assess dryness of the mouth in older people with impaired comprehension by conventional methods such as the chewing gum or Saxon test, which require cooperation. The moisture meter used here, however, allows measurements to be made in only approximately 2 seconds, making it suitable for use in such an elderly population, as has already been reported elsewhere $^{11)}$. The total number of microbes on the tongue surface was measured using a rapid oral bacteria detection device (Bacterial Counter DU-AA01NP-H; Panasonic Health Care, Tokyo, Japan). This device was developed for a technique known as DiElectroPhoretic Impedance Measurement (DEPIM), which is based on dielectrophoresis and the measurement of impedance. Samples were collected using a sterilized swab and a constant-pressure sampler, which prevents exertion of pressure exceeding $20 \mathrm{gf}$ on sampling. Before sampling, preliminary moistening was performed by spraying the tongue surface 
Tongue Coating Record (TCR)

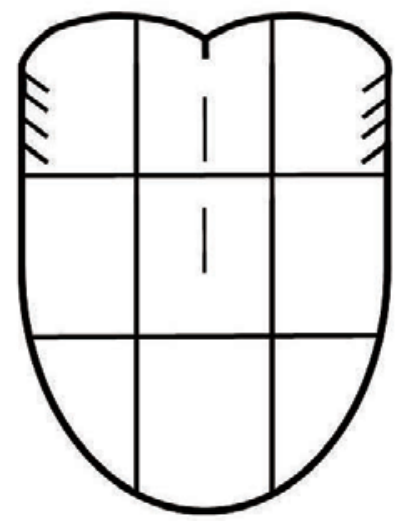

Name :

Date :

Score 0 : Tongue coating not visible.

Score 1 : Tongue coating thin, papillae of tongue visible.

Score 2 : Tongue coating very thick, papillae of tongue not visible.
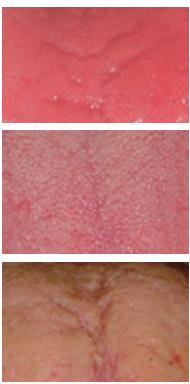

Tongue Coating Index (TCI)

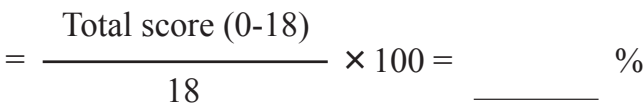

Fig. 1 Tongue Coating Record (TCR) form and method of calculation for Tongue Coating Index (TCI)

Table 1 Correlation between tongue coating status and each factor

\begin{tabular}{|c|c|c|c|c|}
\hline Factors & Mean & $\mathrm{SD}$ & $\mathrm{r}_{\mathrm{s}}$ & $\mathrm{p}$ \\
\hline Moisture level of the tongue & 25.5 & 6.7 & 0.006 & 0.968 \\
\hline Total anaerobic bacteria on tongue surface & $2.01 \times 10^{7}$ & $2.0 \times 10^{7}$ & 0.449 & 0.003 \\
\hline Type of food for grain dish & \multicolumn{2}{|c|}{$\begin{array}{l}\text { Tube feeding: } 3 \text { people } \\
\text { Paste diet: } 8 \text { people } \\
\text { Whole porridge: } 0 \text { people } \\
\text { Soft rice: } 11 \text { people } \\
\text { Regular diet: } 19 \text { people }\end{array}$} & 0.286 & 0.07 \\
\hline Type of food for side dish & \multicolumn{2}{|c|}{$\begin{array}{l}\text { Jelly diet: } 4 \text { people } \\
\text { Paste diet: } 4 \text { people } \\
\text { Little grainy paste diet: } 3 \text { people } \\
\text { Chopped diet: } 22 \text { people } \\
\text { Regular diet: } 8 \text { people }\end{array}$} & 0.388 & 0.012 \\
\hline Various levels of care & \multicolumn{2}{|c|}{$\begin{array}{l}\text { Requiring support: } 3 \text { people } \\
\text { Requiring long-term care1: } 1 \text { person } \\
\text { Requiring long-term care2: } 5 \text { people } \\
\text { Requiring long-term care3: } 6 \text { people } \\
\text { Requiring long-term care4: } 14 \text { people } \\
\text { Requiring long-term care5: } 12 \text { people }\end{array}$} & -0.308 & 0.05 \\
\hline Sex & \multicolumn{2}{|c|}{5 men, 36 women } & -0.128 & 0.424 \\
\hline
\end{tabular}




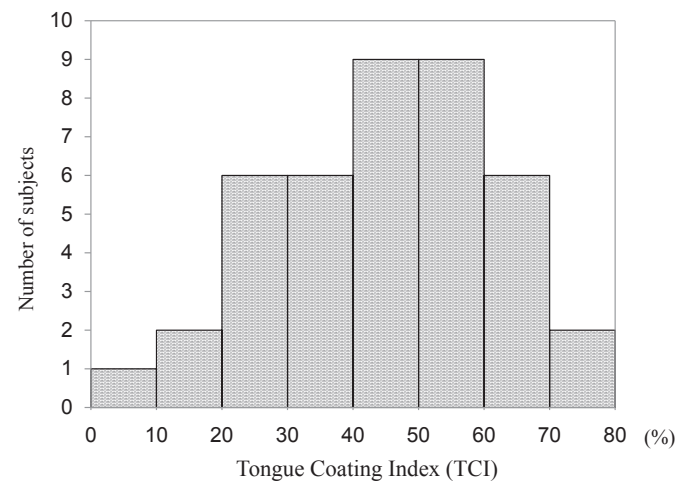

Fig. 2 Number of participants and tongue coating status

with approximately $2 \mathrm{ml}$ of water. The sterilized swabs were dipped in tap water in advance. Samples were obtained by swabbing anteriorly 5 times, commencing from the anterior area of the terminal sulcus on the median groove of the tongue. After sampling, the used swab was inserted into a disposable cup containing pure water and the total number of oral microbes in the sample measured. As this device is portable, measurement can also be performed at the bedside in approximately 2 minutes, which is shorter than the time required for measurement by the culturing method. Therefore, it is considered suitable for sampling in individuals who cannot be easily transported due to care needs, such as those targeted in the present study. In addition, this method was adopted here as it has shown high reliability, as indicated by strong correlations with oral bacterial and microbial counts obtained by culturing and fluorescent microscopic methods ${ }^{3}$. Food type, care level, and sex were determined by a questionnaire given by attending nurses. An ordinal scale was used based on the food type of the grain dish, the food type of the side dish, and level of care. The food type of the grain dish (staple dish) was classified as tube feeding $(=1)$, paste $(=2)$, gruel (rice: water $=1: 5)(=3)$, soft $(=4)$, or regular $(=5)$; that of the side dish was similarly classified as jelly as supplementary food $(=1)$, paste $(=2)$, small-grain
Table 2 Statistical analysis of tongue coating status

\begin{tabular}{cccc}
\hline \hline Mean & SD & Median & Range \\
\hline 43.7 & 16.8 & 44.4 & $5.6-77.8$ \\
\hline
\end{tabular}

paste $(=3)$, chopped $(=4)$, or regular $(=5)$. Care needs were graded as support $(=1)$, level $1(=2)$, level $2(=3)$, level $3(=4)$, level $4(=5)$, or level $5(=6)$. Correlations between tongue coating status and each factor were analyzed using Spearman's rank correlation coefficient. The participants were divided into 2 groups according to the median value of the tongue coating index score. Binomial logistic regression analysis (simultaneous entry) was performed using group as the objective variable and factors related to each group as explanatory variables $(\alpha=0.05)$. The statistical software used was IBM SPSS statistics 22.0 (International Business Machines Corporation, Chicago, IL, USA). This study was approved by the Ethics Committee of Tokyo Dental College (approval no. 453). Written informed consent was obtained from each participant prior to commencement of the study.

\section{Results}

A total of 41 individuals were enrolled in the study (mean age, $87.7 \pm 7.7$ years; 5 men and 36 women). Tongue coating status showed a mean value of $43.7 \pm 16.8$ and median value of 44.4 (5.6-77.8) (Fig. 2, Table 2). Among all participants, 15 showed a value of less than 44.4, while 26 showed a value of 44.4 or above. Table 1 shows the correlations between tongue coating status and the factors evaluated. Tongue coating status showed a correlation with the total number of microbes on the tongue surface $\left(r_{s}=0.449, p=0.003\right)$, food type of the side dish $\left(\mathrm{r}_{\mathrm{s}}=0.388\right.$, $\mathrm{p}=0.012)$, and care level $\left(\mathrm{r}_{\mathrm{s}}=-0.308\right.$, $p=0.050)$. The logistic regression analysis revealed a significant correlation with the 
Table 3 Factors associated with tongue coating status

\begin{tabular}{lccc}
\hline \multicolumn{1}{c}{ Factors } & Odds ratio & 95\%CI & p value \\
\hline Moisture level of the tongue & 1.035 & $0.903-1.186$ & 0.621 \\
Total anaerobic bacteria on tongue surface & 1 & $1.000-1.000$ & 0.075 \\
Type of food for grain dish & 0.332 & $0.074-1.496$ & 0.151 \\
Type of food for side dish & 9.599 & $1.009-91.340$ & 0.049 \\
Various levels of care & 0.702 & $0.325-1.516$ & 0.368 \\
Sex & 0.066 & $0.003-1.645$ & 0.098 \\
\hline
\end{tabular}

food type of the side dish (odds ratio, 9.599; 95\%CI, 1.009-91.340; $\mathrm{p}=0.049$ ) (Table 3).

\section{Discussion}

The results of the present study revealed a correlation between tongue coating status and the total number of microbes on the tongue surface $\left(\mathrm{r}_{\mathrm{s}}=0.449, \mathrm{p}=0.003\right)$. The mean number of microbes on the tongue surface was $2.0 \times 10^{7} \mathrm{CFU} / \mathrm{ml}$, which was higher than that previously reported in healthy older people $^{10)}$. It has become clear that the higher the tongue coating status, the greater the total number of microbes on the tongue surface ${ }^{12}$, and that tongue coating status affects the number of anaerobic bacteria in saliva ${ }^{9}$. Furthermore, the profile of the microbial flora on the tongue surface has been reported to be similar to that in saliva ${ }^{4,10)}$. These findings suggest that the microbial count in the saliva observed in the present study is likely to be higher than that in healthy older people. Here, the mean moisture level of the tongue surface was less than 27 , which is considered a criterion for dry mouth $^{3)}$, which was indeed present in many of the participants. The total number of microbes on the tongue surface showed an increase with an increase in the tongue coating index score, which is in agreement with the results of earlier studies ${ }^{14)}$. Tongue coating status showed a correlation with the food type of the side dish $\left(r_{\mathrm{s}}=0.388\right.$, $p=0.012)$. Jelly and paste have low adhesiveness and high aggregability, which means bolus formation is easier with this type of food. On the other hand, paste is more adhesive than small-grain paste, and chopped food is more likely to be retained in the oral cavity, which may influence tongue coating status. The dorsum of the tongue is covered by 4 types of lingual papillae: filiform, fungiform, foliate, and circumvallate ${ }^{6)}$. Because of these lingual papillae, the dorsum of the tongue shows fine convexities and concavities compared with other areas of the oral mucosa, and this contributes to the deposition of filth and proliferation of microorganisms in deep areas, which present anaerobic conditions.

The amount of time required before the bolus is transferred to the pharynx from the oral cavity is affected by the physical properties of the food being consumed. As the hardness of the food increases, compression grinding gives way to biting, thus prolonging retention time ${ }^{1)}$. This suggests that the chopped food and small-grain paste used in the present study would also have affected tongue coating status. Here, grain showed no correlation with the tongue coating index score, possibly because consumption of grain was lower than that of the side dishes.

Tongue coating status showed a correlation with care level $\left(\mathrm{r}_{\mathrm{s}}=-0.308, \mathrm{p}=0.050\right)$. Assistance is necessary in maintaining oral hygiene in older people with care needs, as they have difficulty in performing such cleaning by themselves. Because of problems such as a shortage of care givers, however, it is difficult to provide sufficient oral cleaning frequently enough and for a long enough duration ${ }^{7}$. 
Therefore, when an elderly person is considered to have need for only partial assistance or support, they are considered to be capable of maintaining oral hygiene by themselves. This is not always the case, however, and this can result in an increase in the tongue coating due to inadequate cleaning.

The results of the present logistic regression analysis revealed a correlation between tongue coating status and type of food in the side dish. One study reported a low level of autonomic nerve activity in individuals on a jelly diet, which can be swallowed without mastication or grinding, but a high level in individuals on a soft diet such as gruel, mashed potatoes, or well-cooked carrot $^{8}$. Another study reported lower tongue pressure in elderly people eating a soft food as their grain dish as well as their side dish than in those eating a soft food as their grain dish and ordinary food as part of their side dish ${ }^{13}$. Taken together, these findings show that side dishes requiring tongue movement will compensate to some extent for an absence of foods that require chewing, and thus help maintain oral function, including oral hygiene.

As populations age worldwide, the number of older people requiring nursing care is likely to increase. Given this trend, the importance of prevention of aspiration pneumonia, a major cause of death in older people, is expected to increase. Care givers are not qualified to judge which type of food is best suited to the elderly person in their care. This means that older people are sometimes given an unnecessarily soft diet, which may result in a deterioration in mastication, swallowing, oral function, and oral hygiene, which in turn may lead to anorexia. Many organs and tissues are involved in the stomatognathic system, which is responsible for mastication. Mastication is considered confer many advantages, in addition to nutritional intake: it stimulates sense of taste; increases saliva secretion; improves self-cleaning of the mouth; and increases cerebral blood flow. The results of the present study support these favorable effects of mastication. Dentists and dental hygienists are encouraged to individually assess mastication ability and determine the appropriate food type in promoting mastication.

\section{Conclusion}

The present results demonstrated a correlation between the status of the tongue coating and the type of food consumed in elderly individuals resident in a care home.

\section{Conflict of Interest}

The authors report no conflict of interest directly relevant to the content of this article.

\section{References}

1) Arai E, Yamada Y (1993) Effect of the texture of food on the masticatory process. Jpn J Oral Biol 35:312-322.

2) Hamada S, Slade HD (1980) Biology, immunology, and cariogenicity of Streptococcus mutans. Microbiol Rev 44:331-384.

3) Kikutani T, Tamura F, Takahashi Y, Konishi K, Hamada R (2012) A novel rapid oral bacteria detection apparatus for effective oral care to prevent pneumonia. Gerodontology 29: e560-e 565 .

4) Mager DL, Ximenez-Fyvie LA, Haffajee AD, Socransky SS (2003) Distribution of selected bacterial species on intraoral surfaces. J Clin Periodontol 30:644-654.

5) Nolte WA (1977) Oral Microbiology, pp.214215, Mosby, Philadelphia.

6) Norton NS (2014) Netter's Head and Neck Anatomy for Dentistry, 2nd ed., pp.379-380, Saunders, Philadelphia.

7) Ogami K, Okada C, Tasaka A, Ogiwara T, Ueda T, Sakurai K (2010) Oral health care awareness of staffs in hospitals and nursing health care facilities for the elderly. J J Gerodont 25: 26-30.

8) Ohta M, Ueda T, Sakurai K (2017) Effect of chewing or compressing food on autonomic nervous activity in older adults. Gerodontology 34:434-440.

9) Ryu M, Ueda T, Saito T, Yasui M, Ishihara K, Sakurai K (2010) Oral environmental factors affecting number of microbes in saliva of com- 
plete denture wearers. J Oral Rehabil 37: 194-201.

10) Sachdeo A, Haffajee AD, Socransky SS (2008) Biofilms in the edentulous oral cavity. J Prosthodont 17:348-356.

11) Saito M, Ono Y, Kitamura N, Yamaguchi M, Saito C (2008) A study of moisture content in oral mucosa in the elderly. Part I. Evaluating the precision of oral moisture checking devices. Ann Jpn Prosthodont Soc 23:90-96.

12) Shimizu T, Ueda T, Sakurai K (2007) New method for evaluation of tongue-coating status. J Oral Rehabil 34:442-447.

13) Tsuga K, Shimada M, Kuroda R, Hayashi R, Yoshikawa M, Sato K, Saito N, Yoshida M, Maeda Y, Kida O, Akagawa Y (2005) The relationship between tongue pressure and selected meal form of elderly people taking "Geriatric Soft Food". J J Dysphagia Rehabil 9: 56-61.

14) Ueno T, Tei K, Orui S, Nakagawa R, Yasumi N, Izumiyama Y, Totsuka Y (2000) A study on oral bacterial flora with relation to general health (I). The oral bacterial flora of elderly nursing home patients. JJ Gerodont 14:265-269.

15) Yaegaki K, Sanada K (1992) Biochemical and clinical factors influencing oral malodor in periodontal patients. J Periodontol 63:
783-789.

16) Yasui M, Ryu M, Sakurai K, Ishihara K (2012) Colonisation of the oral cavity by periodontopathic bacteria in complete denture wearers. Gerodontology 29:494-502.

17) Yoneyama T, Yoshida M, Horus T, Mukaiyama H, Okamoto H, Hoshiba K, Ihara S, Yanagisawa S, Ariumi S, Morita T, Mizuno Y, Ohsawa T, Akagawa Y, Hashimoto K, Sasaki H; Oral Care Working Group (2002) Oral care reduces pneumonia in older patients in nursing homes. J Am Geriatr Soc 50:430-433.

18) Yoneyama T, Yoshida M, Matsui T, Sasaki H (1999) Oral care and pneumonia. Oral Care Working Group. Lancet 354:515.

\section{Correspondence:}

Dr. Koichiro Ogami

Department of Removable

Prosthodontics and Gerodontology,

Tokyo Dental College,

2-9-18 Kanda-Misakicho, Chiyoda-ku,

Tokyo 101-0061, Japan

Tel: + 81-3-6380-9201,

Fax: + 81-43-270-3935

E-mail: ogami@tdc.ac.jp 